\title{
Socioepistemología y Empoderamiento: la profesionalización docente desde la problematización del saber matemático
}

\author{
Socioepistemology and Empowerment: teacher professionalization from \\ problematization of mathematical knowledge
}

\author{
Daniela Reyes-Gasperini ${ }^{*}$ \\ Ricardo Cantoral $^{* *}$
}

\begin{abstract}
Resumen
La Matemática Educativa, como disciplina académica, se propone un objetivo mayor: democratizar el aprendizaje de las matemáticas. Para alcanzarlo, habrá que transitar de una perspectiva platónica, centrada en objetos abstractos ajenos a la realidad, hacia una visión socioepistemológica que asume a las prácticas sociales como la base de la construcción del conocimiento matemático de toda persona. Esto precisa de numerosas restructuraciones del sistema educativo, una de ellas es la profesionalización docente. En este artículo, postulamos al empoderamiento como el proceso que atiende a la profesionalización docente desde una mirada socioepistemológica a través de la problematización del saber matemático escolar.
\end{abstract}

Palabras-clave: Empoderamiento Docente. Socioepistemología. Problematización del saber. Proporcionalidad.

\begin{abstract}
Mathematics Education, as an academic discipline, has a main goal: to democratize mathematics learning. To achieve this, it is necessary to move from a platonic perspective on abstract objects outside of reality, to a socioepistomological point of view which assumes social practices are the bases for construction of mathematical knowledge for all people. This requires numerous restructurings of the educational system, one of which is teacher professionalization. In this paper, we postulate empowerment as the process serving teacher professionalization from a socioepistomological point of view from the problematization of school mathematical knowledge.
\end{abstract}

Keywords: Teacher empowerment, socioepistemology, problematization of knowledge, proportionality.

\section{Introducción}

La función docente, en un sentido amplio, suele ser cuestionada por sectores de la sociedad contemporánea. Es usual, por ejemplo, que se ponga en duda el desempeño del

\footnotetext{
* Maestra en Ciencias, especialidad Matemática Educativa y actual Doctorante, ambas en el Centro de Investigación y de Estudios Avanzados del IPN, Cinvestav-IPN, Distrito Federal, México. Dirección Postal: Av. Instituto Politécnico Nacional, 2508, Col. San Pedro Zacatenco, 07360, Distrito Federal, México. E-mail: dreyes@ cinvestav.mx

** Doctor en Ciencias, especialidad Matemática Educativa por el Centro de Investigación y de Estudios Avanzados del IPN, Cinvestav-IPN. Jefe del Departamento de Matemática Educativa del Centro de Investigación y de Estudios Avanzados del IPN, Cinvestav-IPN, Distrito Federal, México. Dirección Postal: Av. Instituto Politécnico Nacional, 2508, Col. San Pedro Zacatenco, 07360, Distrito Federal, México. E-mail: rcantor@cinvestav.mx
} 
docente desde diversos ámbitos sociales: la familia, la prensa, el gobierno y desde muchos otros espacios profesionales. Nosotros, en cambio, partimos del reconocimiento del docente como intelectual profesional que se ocupa de la formación académica, ética y ciudadana de la juventud.

El creciente interés por la formación y profesionalización docente en el campo de las matemáticas, y su repercusión en distintos regiones del orbe, es constatado por la gran diversidad de publicaciones (BALL; THAMES; PHELPS, 2008; CANTORAL, 2013a; CLIMENT et al., 2013; CONTRERAS et al., 2012; DA PONTE; QUARESMA; BRANCO, 2012; GELLERT; BECERRA; CHAPMAN, 2013; OLIVEIRA ARAMAN; BATISTA, 2014; PARADA; PLUVINAGE, 2014; LLINARES; VALLS; ROIG, 2008; RIVAS; GODINO; CASTRO, 2012).

Las investigaciones en esta temática se sustentan normalmente en reflexiones que denominaremos clásicas: estudios sobre concepciones, creencias, formación continua o profesionalización docente; sobre el contenido pedagógico del conocimiento, el contenido del conocimiento para la enseñanza; sobre las prácticas de los docentes a través del análisis de tareas propuestas, el tipo de discurso en el aula y los roles asumidos por los docentes y estudiantes; conocimientos teóricos y prácticos que deben tener los docentes y, así, un largo etcétera. Es decir, existe una fuerte centración en los procesos didáctico-pedagógicos, sin realizar una problematización del saber matemático escolar, es decir, hacer del saber un problema, un objeto de análisis didáctico, localizando y analizando su uso y su razón de ser. Esto último, consideramos, se ubica en el núcleo de la acción didáctica: le llamaremos el estudio de la naturaleza ontológica y epistemológica del saber matemático enseñando. Nuestra contribución reside, específicamente, en este asunto: atender a la profesionalización docente desde la problematización del saber matemático.

Mediante la teoría Socioepistemológica (CANTORAL, 2003, 2011, 2013b; CANTORAL; FARFÁN, 1998; CANTORAL; TUYUB, 2012; SOTO; CANTORAL, en prensa; MONTIEL; JÁCOME, en prensa) afirmamos, a contracorriente de lo que suele afirmarse, que es en el propio discurso Matemático Escolar (dME) donde radica el mayor conflicto de la enseñanza y el aprendizaje de las matemáticas y de sus aspectos didácticos. En este sentido, hablemos de algunas características del $d M E$. La matemática escolar, producto de una trasposición didáctica (CHEVALLARD, 1999), lleva al saber sabio hacia el saber enseñado; es decir, el saber matemático sufre modificaciones adaptativas, de forma progresiva, con el fin de seleccionar, organizar y estructurar los conocimientos matemáticos que serán incluidos en las unidades temáticas de la escuela. Asimismo, es sabido que la 
manera de abordar la matemática en el sistema educativo ocurre mediante la centración en los objetos matemáticos, concebidos estos como entidades abstractas que son ejemplificadas y ejercitadas; eludiendo en el tratamiento didáctico la construcción del conocimiento matemático por parte del estudiante, esto es, se concibe que las matemáticas tratan con objetos abstractos, anteriores por tanto a la praxis social y, en consecuencia, externas al individuo, siendo el profesor quien comunica verdades preexistentes a sus alumnos, normado por el $d M E$ (CANTORAL, 2003). Por tanto, la construcción social del conocimiento matemático queda rezagada en el $d M E$.

De este modo, como el saber enseñando es producto de la transposición didáctica y, por tanto, sufre transformaciones, la Socioepistemología propone modificaciones al nivel del $d M E$, su rediseño ( $r d M E)$, a través de plantearse el estudio de la construcción social del conocimiento matemático para atender al cuestionamiento del qué se aprende.

Ahora bien, ante el planteamiento de una nueva mirada hacia la matemática que la construcción social de los conocimientos matemáticos, ¿cómo repercute esto entre los docentes en etapa de profesionalización? ¿Qué rol juega el saber matemático en dicho proceso formativo? Mientras que en los enfoques clásicos se cuestionan cuánto y cuál es el tipo de conocimiento necesario del profesor para la enseñanza, cuáles son las mejores estrategias didácticas para llevar al aula y hacer más accesible un saber matemático, este enfoque se cuestiona ¿cuál es el proceso que debe vivir el saber matemático para que la visión del aprendizaje esté centrada en prácticas sociales y no en objetos abstractos ajenos a la realidad?, ¿qué proceso debe vivir el docente para que logre la apropiación del saber matemático escolar y logre hacerse dueño de su práctica? Desde la teoría Socioepistemológica atendemos a esta problemática afirmando: es indispensable el empoderamiento docente para hacerse dueño de su propia práctica a través de la problematización del saber matemático escolar (psme) y, así, promover un cambio significativo en su práctica docente y una mejora en la educación y la didáctica de la matemática.

En este artículo presentamos un estudio de caso mediante un análisis cualitativointerpretativo de la práctica de un profesor de escuela media (12-15 años) del sistema escolar mexicano, para lo cual hemos construido una unidad de análisis socioepistémica con base en las dimensiones social, epistemológica, cognitiva y didáctica de la proporcionalidad en matemáticas, que nos permitió construir una estructura teórica para evidenciar el cambio de práctica de un docente, producto del cambio de relación al saber matemático a través de la psme. Este cambio de visión en cuanto al aprendizaje de la matemática, a partir del cambio de relación al saber matemático, transita de una mirada platónica del conocimiento que 
impregna por igual al quehacer didáctico de nuestros días en donde un profesor comunica verdades preexistentes a sus alumnos, mediante un discurso basado en objetos matemáticos, hacia una mirada socioepistemológica en donde se privilegie la validación de las distintas argumentaciones, se permita la emergencia de las diversas racionalidades contextualizadas, se posea un carácter funcional del saber, se favorezca una resignificación progresiva, considerando varios marcos de referencia, considerando en la base de la construcción del conocimiento, a las prácticas sociales. El estudio de caso nos permitirá evidenciar, caracterizar y ejemplificar el fenómeno del empoderamiento docente.

Como resultado de esta investigación postulamos como hipótesis que, por un lado, una propuesta de innovación para los diseños de formación docente debe sustentarse en la psme más que en la producción de prototipos de herramientas didácticas para el aula; por el otro, que la construcción de una unidad socioepistémica con base en la problematización del saber matemático ( $\mathrm{psm}$ ) desde un estudio socioepistemológico permitirá, en un futuro, evaluar la inmersión a un proceso de empoderamiento docente considerando a la psme como punto de partida; y, por último, postulamos al empoderamiento docente como un mecanismo para atender al fenómeno de exclusión de la construcción del conocimiento matemático provocado por el $d M E$ (SOTO, 2010) que debe acompañar el rediseño e inserción en el sistema educativo de este último.

\section{Problema y marco teórico}

Como se dijo anteriormente, en estos últimos años la profesión docente comenzó a ser estudiada a profundidad en el campo de la Matemática Educativa (CANTORAL, 2013a; GELLERT; BECERRA; CHAPMAN, 2013). Uno de los factores a los cuales se enfrenta un docente, habitualmente, es a los cambios educativos ya sean reformas educativas en cuanto a programas de estudio, cambio de libros de texto, cambio de paradigma de enseñanzaaprendizaje, entre otros. Es por esto que gran parte de las investigaciones estudian cómo atender a esos cambios en el momento indicado. Es necesario aclarar, que dichos estudios y sus posibles propuestas de cambio estarán enmarcadas en el contexto desde el cual se realiza el estudio, pues reconocemos nuestra identidad latinoamericana al momento de realizar la investigación (SILVA-CROCCI; CORDERO OSORIO, 2013).

En particular, la Teoría Socioepistemológica plantea que el estudio de la naturaleza del saber matemático y la posterior psme por parte del docente le brindará al profesor la 
oportunidad de transformar su realidad y tomar decisiones sobre sus acciones didácticas a través de una herramienta principal en su profesión: el saber matemático escolar.

Desde sus inicios, la Socioepistemología se cuestionó qué es lo que se está enseñando, qué tipo de saber matemático está viviendo en el sistema educativo, a quién, para qué y por qué debe ser enseñado, aunado al cómo se deberían enseñar los contenidos matemáticos. Dado este cuestionamiento, fue necesario cambiar la centración: dejar de observar al concepto matemático en sí, y comenzar a observar las prácticas que lo producen o favorecen su necesidad. Para este análisis la Socioepistemología incorpora una nueva componente a las cognitiva, didáctica y epistemológica de la Matemática Educativa: la componente social; e integra las cuatro dimensiones de manera tal que logra una mirada sistémica de los fenómenos a abordar. El problema de la enseñanza y aprendizaje no se reduce más al fácil acceso de las explicaciones didácticas o a rellenar los agujeros de contenidos matemáticos entre estudiantes y profesores; el problema, en este momento, se transforma hacia el cuestionamiento del saber matemático escolar inmerso en la vida del sistema didáctico.

Se ha puesto en evidencia que el $d M E$ está centrado en objetos matemáticos que se estudian a través de la incorporación de ciertos algoritmos, argumentaciones y procedimientos específicos, y sobre todo, que carece de un sentido humano, es decir, en este tipo de centración la matemática es preexistente a cualquier actividad humana. Entonces, entendiendo que el $d M E$ excluye de la construcción del conocimiento matemático (SOTO, 2010; SOTO; CANTORAL, en prensa), que los docentes también han sido excluidos de ésta (REYESGASPERINI, 2010; REYES-GASPERINI; CRESPO, 2011) y que la puesta en práctica de las herramientas que de la investigación surgen, aun con la apropiación de ellas, no es un hecho trivial (LEZAMA, 2005) ¿cuál es el proceso que debe vivir el saber matemático para que la visión del aprendizaje esté centrada en prácticas sociales y no en objetos abstractos ajenos a la realidad postulados por el $d M E$ ?, ¿qué proceso debe vivir el docente en el cual logre la apropiación del saber matemático escolar para hacerse dueño de su práctica, y pueda sumarse a esta nueva visión de la matemática? Nuestras respuestas son las siguientes: se debe cuestionar y problematizar el saber matemático con el fin de poder construir una unidad que permita la psme para discutir junto al docente la esencia de los saberes matemáticos escolares.

Así, el docente comenzará a vivir un proceso de empoderamiento en donde la problematización le permitirá hacerse dueño de su propia práctica. Entonces, con base en esta reflexión nos vimos en la tarea de estudiar y caracterizar al proceso de empoderamiento docente como uno de los mecanismos didácticos que acompañe al $r d M E$, para potenciar el 
aprendizaje con base en la construcción social del conocimiento, y así, atender a la exclusión que provoca el $d M E$ actual (REYES, 2011; REYES-GASPERINI, CANTORAL, 2012).

A continuación, se mostrarán los fundamentos teóricos que hemos construido para analizar los datos obtenidos del estudio de casos. En primer lugar, se estudian las distintas corrientes que han atendido el fenómeno social del empoderamiento y se extraen las características principales que permiten postular qué se entiende por empoderamiento docente desde una visión socioepistemológica. En segundo lugar, se construye una unidad de análisis, a la que hemos denominado unidad socioepistémica, a partir de la psm con base en las dimensiones social, epistemológica, cognitiva y didáctica, de la noción de la proporcionalidad, como herramienta que permita analizar las interacciones didácticas del docente.

\subsection{Empoderamiento docente}

Con el fin de caracterizar al fenómeno del empoderamiento docente, se analizó lo que las distintas comunidades de conocimiento asumen sobre el fenómeno de carácter social denominado empoderamiento y, desde allí, se construyó la correspondiente a nuestra disciplina, la Matemática Educativa. Desde un enfoque psicosocial (MARTÍN MARURI, 2011), social (SILVA DREYER; MARTÍNEZ GUZMÁN, 2007), feminista (CAMACHO, 2003), desde la Psicología Comunitaria (MONTERO, 2006), o bien, desde un enfoque educativo (HOWE; STUBBS, 1998, 2003; STOLK et al., 2011), se encuentran elementos transversales que caracterizan al empoderamiento: se entiende como un proceso del individuo en colectivo, que parte de la reflexión para consolidarse en la acción, que se produce desde el individuo sin la posibilidad de ser otorgado y, por sobre todas las cosas, que transforma su realidad.

En particular, los proyectos que tienen como objetivo impulsar el empoderamiento docente (HOWE; STUBBS, 1998, 2003; STOLK et al., 2011) se focalizan en darle al docente herramientas para que realicen nuevas situaciones para el aula, poniendo como punto importante la contextualización, ya sea mediante el conocimiento (conocer que existe) de nuevas investigaciones relacionadas con el tema a abordar, como así también, mediante la muestra de situaciones que brinden un contexto a lo que ellos ya conocen. Todo con el objetivo de que obtengan una actitud de liderazgo, confianza y mejora en sus prácticas para la enseñanza, enfatizando el hecho de que adquieran el poder de tomar las riendas de su propio crecimiento. 
Sin embargo, si bien nosotros coincidimos plenamente con los resultados que se esperan, consideramos que este tipo de análisis se reduce a una interpretación pedagógica. Dado el carácter socioepistemológico que se añade a este fenómeno social, la problematización del saber matemático escolar (psme) fungirá como factor imprescindible para el empoderamiento docente. Partimos de los contenidos fundamentales para el sistema didáctico dependiendo del nivel donde se realice el estudio, en este caso, el nivel secundario. Asimismo, entendemos la psme como la acción que parte de la introspección, la mirada del que aprende y los usos que este saber posee en la cotidianeidad, apoyándose en las discusiones y reflexiones colectivas y en las investigaciones sobre dicho saber, o bien, siendo ellos mismos quienes se adentren a tal investigación. Esta psme permitirá el reconocimiento y validación de distintas argumentaciones, la emergencia de las diversas racionalidades contextualizadas, el entendimiento del carácter funcional del saber, la resignificación progresiva considerando varios marcos de referencia, considerando en la base de la construcción del conocimiento a las prácticas sociales.

Para lograr una psme debemos, con anterioridad, realizar un estudio sobre la problematización del saber matemático (psm). Ésta refiere al hecho de hacer del saber un problema, un objeto de análisis didáctico, localizando y analizando su uso y su razón de ser: se analiza la naturaleza del saber (dimensión epistemológica); el uso del saber (dimensión social); apropiación del saber (dimensión cognitiva) y la difusión del saber (dimensión didáctica).

\subsection{Unidad socioepistémica: el caso de la proporcionalidad}

Dado que el ejemplo de la proporcionalidad surge de la observación empírica, se acude a realizar una revisión de las investigaciones que la comunidad científica de Matemática Educativa realizó al respecto y a la literatura pertinente que nos ayudará a confeccionar un cuerpo teórico que denominamos unidad de análisis socioepistémica sobre la noción de proporcionalidad con el fin de poder, mediante el análisis de la relación que posee el docente con este saber matemático, identificar elementos que nos permitan postular evidencias sobre el fenómeno que nos compete: el empoderamiento docente. La construcción de dicha unidad tiene una estructura sistémica sobre los modelos del pensamiento proporcional, con base en las dimensiones epistemológica, cognitiva, didáctica y social. 


\subsubsection{Dimensión social}

La dimensión social nos permite postular que los conocimientos abstractos y aislados de la realidad, en este caso la noción de proporcionalidad, que en los sistemas educativos están centrados en algoritmos del tipo reducción a la unidad, regla de tres simple o encontrar el cuarto valor faltante, no sólo están asociados a abstracciones como lo presenta el $d M E$, sino que tienen una dimensión práxica, funcional, situacional, histórica que está al nivel de la actividad, y esta dimensión está soslayada por el $d M E$. La dimensión social de nuestra unidad socioepitémica nos lleva a entender a la matemática como parte de la cultura, como producto de la actividad humana.

Entonces, bajo la mirada socioepistemológica, al concebir que los conocimientos se dotan de significados a través de su uso y su funcionalidad, planteamos la necesidad de que docentes y estudiantes, aunque inmersos en un sistema educativo, se relacionen con el saber matemático a fin que construyan las ideas fundamentales sobre dicho saber, más allá de las abstracciones, procedimientos y el aprendizaje de su aplicación. Es decir, la significación que construirá a partir de la actividad de relacionarse al saber matemático como aquel que es producto de la cultura, le permitirá entender aquellas nociones que, las miradas platónicas, consideran como la matemática escolar. Por ejemplo, como se dijo anteriormente, la noción de proporcionalidad no recae en la regla de tres simple o la reducción a la unidad, detrás de ello está la idea germinal como comparación de magnitudes, o bien, se reconoce que hay distintos pensamientos que subyacen a la construcción del pensamiento proporcional, lo cual, en el sistema educativo está totalmente soslayado, ya sea en los procesos de formación docente o en la propia interacción áulica.

En síntesis, esta mirada social hacia la matemática nos conducirá a hacer un análisis epistemológico, didáctico y cognitivo partiendo de la base de que los conocimientos matemáticos son productos de las prácticas sociales.

\subsubsection{Dimensión epistemológica}

Si bien fue Eudoxo de Cnidos (390 A. N. E. - 337 A. N. E.), filósofo, astrónomo, matemático y médico griego, discípulo de Platón, quien trabajó con la teoría de proporciones, se reconoce que fue Euclides quien reunió los aportes hechos por él en su célebre Los Elementos. Con el fin de comprender la proporcionalidad, hemos realizado un análisis axiomático de la teoría de proporciones abordada por Euclides en su Libro V. 
Comenzamos analizando la idea de inconmensurabilidad como generadora de la necesidad de plantear la comparación y relación entre magnitudes.

Se sabe que dos magnitudes son conmensurables cuando existe una unidad de medida común.

Definición 1. Una magnitud es parte de una magnitud, la menor de la mayor, cuando mide a la mayor. Equivalentemente, dos magnitudes son conmensurables si existe una magnitud de medida común.

Ejemplo 1. Dos magnitudes, $\overline{A B}$ y $\overline{C D}$, son conmensurables si existe una magnitud, $\overline{M N}$, que les sirve de unidad a ambas, es decir existen $p, q \in \boldsymbol{N}$ tales que:

$A B=p \times M N$ y $C D=q \times M N$.

Por tanto,

$$
\overline{A B}: \overline{C D}:: p: q
$$

Teorema 1. La diagonal y el lado de un cuadrado, son magnitudes inconmensurables.

Demostración.

Sea el cuadrado $\square A B C D$, con lado $\overline{A B}$ y diagonal $\overline{A C}$. Para probar que diagonal y lado no tienen una unidad de medida común, se hará la demostración por contradicción.

Supongamos que existe $\overline{M N}$, unidad de medida para ambas magnitudes, $\overline{A B}$ y $\overline{A C}$, por tanto existen números naturales $p$ y $q$ tales que $A B=p \times M N$ y $A C=q \times M N$.

De lo cual se sigue,

$$
\overline{A C}: \overline{A B}:: q: p
$$

Por el teorema de Pitágoras $A C^{2}=2 \times A B^{2}$, por tanto $\frac{A C}{A B}=\sqrt{2}$, es decir $\frac{q}{p}=\sqrt{2}$, lo que es una contradicción con la irracionalidad de $\sqrt{2}$.

Ahora bien, si no existe una medida común, ¿cómo se pueden medir estas magnitudes? El problema de medir, fue sustituido, en la Teoría Euclidiana, por el problema de comparar. Esta es la pregunta fundamental que dio origen a la teoría de las proporciones entre magnitudes.

En su definición 6 del Libro V, en el cual aborda la proporcionalidad geométricamente, se llaman proporcionales a las magnitudes que tiene la misma razón, en donde, según su definición 3, la razón es una relación cualquiera entre dos magnitudes homogéneas respecto de su cantidad (EUCLIDES, 1991). Por tanto, se concluye que al decir que la relación guarda la misma razón se pretende resaltar el hecho que a pesar de que cambien los tamaños de las magnitudes, la relación que se establece entre ellas se conserva, es 
decir, la razón se mantiene invariante: constante de proporcionalidad (MARTÍNEZ, GONZÁLEZ, 2008).

Si bien sabemos que el estudio de la proporcionalidad como objeto matemático merece un análisis exhaustivamente mayor al presentado (puede verse OBANDO; VASCO; ARBOLEDA, 2014; OLLER; GAIRÍN SALLÁN, 2013), es hasta aquí lo que se considera suficiente para mostrar en nuestra unidad socioepistémica, ya que podemos concluir del análisis de la dimensión epistemológica de este saber, que la esencia de la proporcionalidad radica en la relación entre magnitudes que se mantiene constante.

\title{
2.2.3 Dimensión cognitiva
}

Si bien nuestra intención radica, principalmente, en profundizar sobre la dimensión cognitiva del docente, consideramos oportuno comprender cómo opera el pensamiento cognitivo humano en general, empezando por los niños. Inhelder y Piaget (1972) realizan un estudio experimental con niños para comprender cómo se desarrolla el pensamiento de lo proporcional, utilizando, entre otros ejemplos, una situación respecto al equilibrio de la balanza. El objetivo fue estudiar cómo se elabora el esquema de proporcionalidad en relación con el problema del equilibrio. Sus conclusiones en cuanto al esquema de las proporciones enuncian:

\begin{abstract}
Conviene recordar en primer lugar que en todos los dominios y no sólo en el caso de nuestras actuales experiencias, la comprensión de las proporciones no aparece antes del nivel III A. Se observa a menudo en los sujetos del subestadio II B la búsqueda de una misma relación en el interior de dos relaciones que se comparan entre sí, pero se concibe que la naturaleza de la relación es aditiva: en vez de la proporción $\mathrm{P} / \mathrm{P}^{\prime}=\mathrm{L}^{\prime} / \mathrm{L}$, se tiene entonces una igualdad de diferencias $\mathrm{P}-\mathrm{P}^{\prime}=\mathrm{L}^{\prime}-\mathrm{L}$. La formación de la idea de proporcionalidad supone pues que en primer lugar, se sustituyan las simples relaciones de diferencia por la noción de la igualdad de productos $\mathrm{PL}=\mathrm{P}^{\prime} \mathrm{L}^{\prime}$. Sin embargo importa además señalar que este pasaje de la diferencia al producto pocas veces se realiza de entrada bajo una formación métrica: por lo general la cuantificación numérica de la proporción se halla precedida por un esquema cualitativo fundado en la noción de producto lógico, vale decir, por la idea de que dos factores que actúan juntos equivalen a la acción de otros dos factores reunidos. (INHELDER, PIAGET, 1977, p. 152, las cursivas son nuestras).
\end{abstract}

Godino y Batanero (2002) enuncian, respecto a uno de los modelos del pensamiento proporcional, denominado modelo aditivo, que si bien estos tipos de estrategias son útiles para enfrentar con éxito ciertos problemas más sencillos, no son válidos en el caso general. Aquí, puede darse como ejemplo el caso de $y=-x$, en la cual no se cumple el pensamiento cualitativo sustentado en a más, más... a menos, menos... 
Carretero (1989), distingue dos tipos de estructuras. Por un lado, aquellas que presentan una relación dada entre magnitudes homogéneas a las que denomina modelo multiplicativo escalar; y por el otro, aquellas que presentan una relación entre magnitudes heterogéneas, denominada modelo multiplicativo funcional. Posteriormente, Lamon (1994, apud MARTÍNEZ, GONZÁLEZ, 2008) realiza también una distinción como estrategias de los estudiantes para hallar el valor faltante de una proporción. Él los denomina modelo inter (correspondiente al modelo multiplicativo escalar) y modelo intra (correspondiente al modelo multiplicativo funcional). El trabajo con los diferentes tipos de estructuras multiplicativas en torno a la adquisición de la noción de la proporcionalidad, le permitió concluir a Carretero que "la división es, evidentemente una operación más difícil que la multiplicación, a pesar de la estructura multiplicativa que subyace" (CARRETERO, 1989, p. 95). De esto concluimos que el modelo aditivo precede al modelo multiplicativo escalar, el cual, es menos complejo que el modelo multiplicativo funcional.

Vergnaud (1990) trabaja sobre la teoría de los campos conceptuales, considerándolos como un conjunto de situaciones la cual se pueda "analizar como una combinación de tareas de las que es importante conocer su naturaleza y la dificultad propia" (VERGNAUD, 1990, p. 140). Respecto a la proporcionalidad, compara los campos conceptuales de las estructuras aditivas (aquellas que precisan una adición, sustracción o combinación de ellas) y las estructuras multiplicativas (aquellas que requieren una multiplicación, división o combinación de ellas). Esto le permite generar una clasificación y análisis de las tareas cognitivas y en los procedimientos que potencialmente son puestos en juego en cada una de ellas. Concluye afirmando que "no es superfluo, por el contrario, resaltar que el análisis de las estructuras multiplicativas es profundamente diferente de las estructuras aditivas" (VERGNAUD, 1990, p. 144).

Dado el análisis de la dimensión cognitiva, se han podido sintetizar los modelos de pensamiento proporcional en el siguiente esquema que muestra la Figura 1, el cual nos brindará una herramienta para analizar las interacciones dialécticas entre el docente y los estudiantes cuando se trabaje la proporcionalidad en el estudio de caso. 


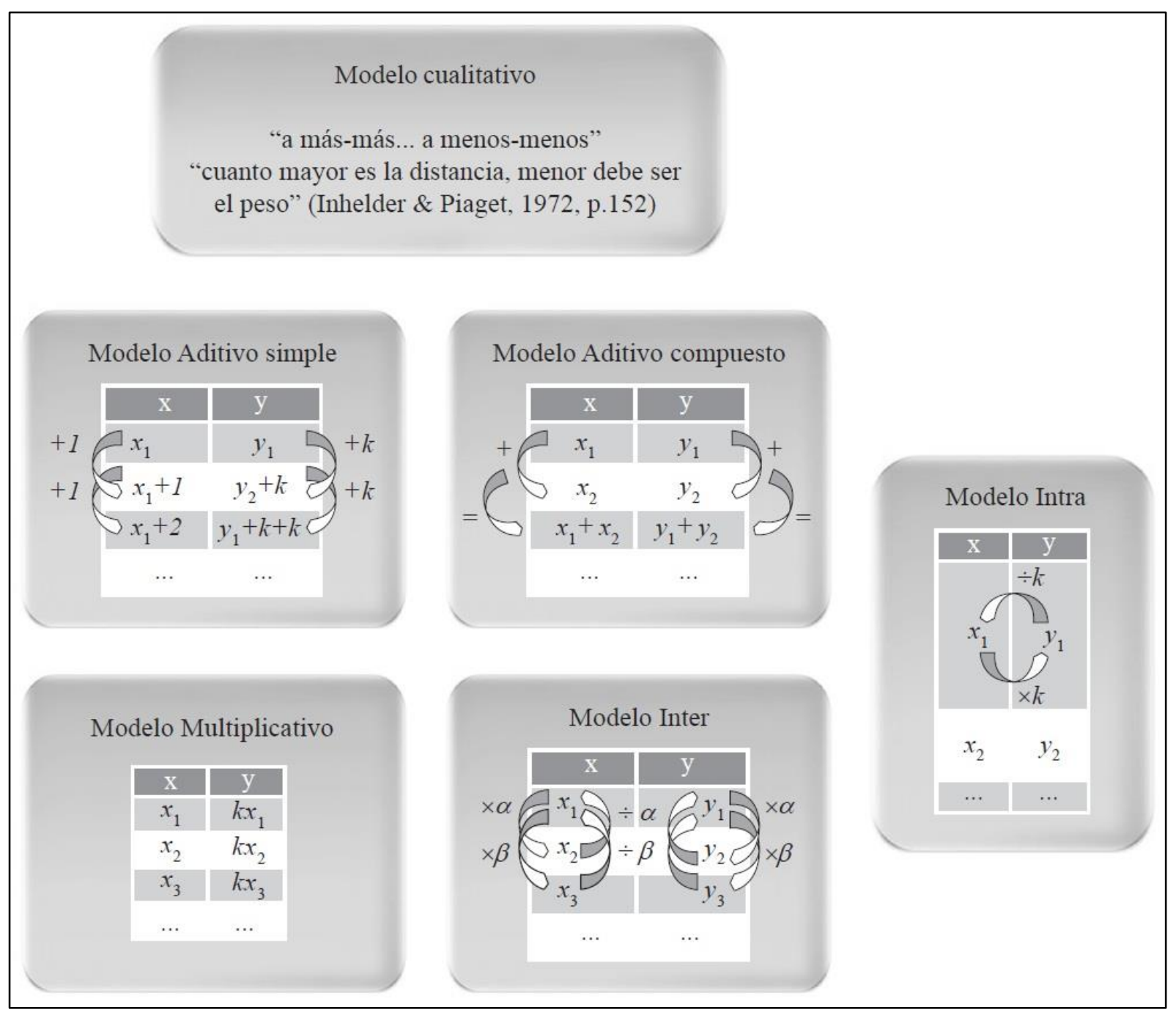

Figura 1 - Modelos del pensamiento proporcional Fuente: desarrollado por los autores

\subsubsection{Dimensión didáctica}

El tema de proporcionalidad es uno de los temas transversales de la educación matemática, por tanto, recaen sobre él varias investigaciones sobre aprendizaje, y, en mayor parte, sobre su enseñanza. Numerosos son los estudios que se realizaron, y realizan actualmente en distintos países, sobre las dificultades de la enseñanza y aprendizaje de la proporcionalidad.

Algunos de estos trabajos evidencian que al trabajar con docentes diseños que tienen como fin superar dificultades respecto al aprendizaje, aunque se reconoce que han avanzado como maestros y tienen acceso a la información que la investigación aporta según el proceso de enseñanza-aprendizaje, ellos no han podido producir modificaciones en su práctica. Es decir, el trabajo sobre los aspectos didácticos y pedagógicos relacionados con la proporcionalidad no basta para generar una transformación en la realidad de los docentes en 
cuanto a su difusión del saber que incorpore los estudios realizados por la investigación. (ITURBE; RUIZ, 2011; SALAZAR; DÍAZ, 2009; VALDEMOROS, 2010).

Otros trabajos analizan las estrategias utilizadas y las dificultades que surgen al resolver problemas sobre la proporcionalidad, ya sea con población estudiantil (OLIVEIRA, 2009; SÁNCHEZ ORDOÑEZ, 2013), profesores en formación (BEN-CHAIM; ILLANY; KERET, 2008; CONTRERAS et al., 2012), o bien, cómo los docentes resuelven e interpretan las resoluciones de estudiantes (RIVAS; GODINO; CASTRO, 2012).

Asimismo, si tomamos algunos libros de texto, podemos observar cómo los pensamientos más destacados en el sistema educativo llegan hasta el pensamiento inter, pero no privilegian un pensamiento intra (BLOCK; GARCÍA, 2009; PISANO, 2011).

En síntesis, la unidad de análisis sociepistémica nos ha permitido entender la esencia de la proporcionalidad como aquella que emerge de la relación entre dos magnitudes, cuya característica es que su relación se mantiene constante. En segundo lugar, que parece no ser suficiente con las reflexiones sobre las didácticas de la enseñanza de razón, fracción y proporción para que los docentes modifiquen sus interacciones dialécticas en cuanto a la relación al saber con sus estudiantes. En tercer lugar, que existen distintos tipos de pensamiento de la proporcionalidad y existen diversas estrategias y dificultades para la resolución de problemas proporcionales. Por último, que es preciso retomar la idea germinal, como producto de la construcción social del conocimiento, presentada sobre la proporcionalidad como la relación que se mantiene constante entre magnitudes para poder resignificar dicho saber, ya que si no, la centración en objetos sólo llevará a la resolución de problemas estandarizados, mas no en el desarrollo del pensamiento proporcional.

\section{Análisis de datos}

Utilizaremos el método de estudio de caso como herramienta de exploración y hallazgo para contribuir a la ampliación, especificación y puntualización de la caracterización del fenómeno de empoderamiento.

El caso será representado por un docente de escuela media (12-15 años) con una experiencia de 15 años frente a grupo en Matemáticas. Formado como Ingeniero Civil en Obras Urbanas, con Maestría en Pedagogía, oriundo de Tijuana, norte de México, participó en un proyecto nacional cuyo objetivo central fue el que los participantes conocieran, profundizaran y compartieran sus conocimientos y prácticas relativas a las matemáticas y las ciencias en el ámbito educativo, a fin de favorecer decisiones relativas al diseño, elaboración 
y análisis de situaciones de aprendizaje para las clases de matemáticas en su relación con otras disciplinas y la vida cotidiana del estudiante. Este proyecto contó con actividades presenciales durante una semana y luego, una actividad a distancia cuya duración fue de poco más que dos meses de trabajo diario.

Su elección estuvo basada en factores de desempeño académico: participación activa durante la fase presencial expresada por los matemáticos educativos a cargo de esta primera fase, compromiso durante la fase a distancia demostrado a través de la participación en los foros de discusión y entrega de trabajos en tiempo y forma, trabajo continuo y de calidad, avances significativos en el examen matemático de ingreso y egreso. Aunado a esto, el docente siempre tuvo plena predisposición para ser observado en sus clases, lo que, metodológicamente, contribuiría en la investigación.

Posterior a ello, se lo observó en sus prácticas de servicio como docente, durante una semana, realizando una inmersión en su vida profesional y personal, con el fin de conocer cuál es la realidad que lo caracteriza. Para ello se realizaron observaciones no participantes de sus clases, entrevistas semiestructuradas, toma escrita de datos, videograbaciones, transcripciones y cuestionarios. Posterior a ello se realizó el análisis de datos.

En este trabajo de campo, se acompañó al docente durante 4 días de labor didáctica, en los cuales se observaron 24 horas reloj de su práctica, de las que, aproximadamente, 20 horas se trabajó sobre la noción de proporcionalidad. En ellas se realizó toma de datos escritos y de 9 horas reloj se realizaron videograbaciones. De toda esta recolección de datos, en este artículo seleccionamos un suceso representativo que funja como evidencia del objetivo de esta investigación: evidenciar, analizar y caracterizar el proceso de empoderamiento docente, para lo cual daremos evidencia de cómo un docente modifica su práctica en cuanto a su relación al saber, a través de la psme, con base en la unidad de análisis socioepistémica.

- Episodio ¿Qué representa el 80?

El problema matemático planteado por el docente, consistía en completar una tabla de valores con casillas vacías. Dos magnitudes heterogéneas (tiempo y distancia) y valores prototípicos sobre los que actuaban los estudiantes:

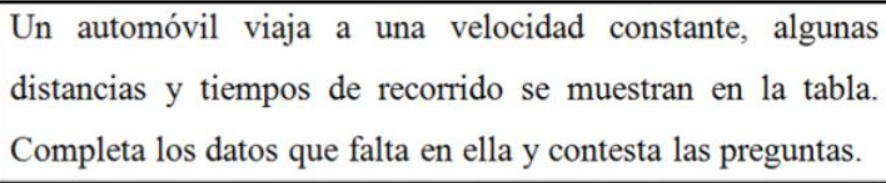

\begin{tabular}{|c|c|c|c|c|}
\hline Tiempo $(\mathrm{h})$ & 1.5 & 3 & 5 & \\
\hline Distancia $(\mathrm{km})$ & & 240 & & 720 \\
\hline
\end{tabular}

Figura 2 - Actividad propuesta por el docente Fuente: desarrollado por los autores 
Una de las interacciones elegida para el estudio fue aquella en que el profesor y sus estudiantes dialogaron como sigue:

[1] Prof: ¿Qué representa el 80? ¡Alguien habló por allá atrás! ¡Esteban! ¿Qué representa el 80 , el valor de qué...?

[2] Esteban: El valor... representa...

[3] Prof: No sabe, ¿verdad?

[4] Esteban: Representa el valor de la proporcionalidad.

[5] Prof: ¿Por qué? ¿Por qué representa el valor de la proporcionalidad?

[6] Esteban: Porque 240 entre 3 es 80.

[7] Ya lo tiene ahí, pero ¿80 qué representa? En valor ¿de qué María?

[8] María: De una hora.

[9] Prof: El valor de una hora. Ponle, una hora, por favor.

En la línea [7], el adverbio pero, el cual se utiliza como enlace que une dos oraciones cuyos significados se contraponen, se restringen o se limitan, y enfatizando nuevamente en la pregunta de ¿qué representa el 80?, da evidencia de que el docente no reconoce la relación entre lo que plantea Esteban en la línea [6] y la noción de constante de proporcionalidad, reflejado en los modelos del pensamiento de la proporcionalidad como un modelo intra. Se denota su aceptación a la respuesta dada por María, lo que muestra que el docente reconoce a la constante de proporcionalidad como aquella que está dada por el procedimiento de reducción a la unidad, lo cual no es un tipo de pensamiento, sino, un algoritmo para encontrar la constante. (Si bien aquí no se muestra a causa del espacio, este método para encontrar la constante de proporcionalidad, la reducción a la unidad, era el más utilizado por el docente durante sus interacciones).

- Episodio problematización del saber matemático escolar

Luego de esta clase, en una hora libre que tuvo el docente, se realizó con él una psme que radicó en cuestionar qué es la proporcionalidad desde situaciones específicas ${ }^{1}$. Lo primero que se le preguntó fue ¿qué significaba proporcionalidad directa? Y el docente contestó que era cuando uno aumentaba y la otra aumentaba o cuando uno disminuye y la otra disminuye (pensamiento cualitativo de la proporcionalidad). Ante su respuesta, se graficó la función $y=x$ y se le preguntó si era una función de proporcionalidad directa y el docente contestó que no, porque una aumentaba y la otra disminuía.

Dada esta respuesta, se le mostró la tabla numérica de la función $y=2.5 x$ (una función que había sido trabajada en clase minutos antes) con valores $(1,2,3)$ en el dominio (ver Figura 3) y se le preguntó por qué él aseguraba que esa función era de proporcionalidad

\footnotetext{
${ }^{1}$ Cabe mencionar que este tipo de interacciones se habían tenido durante el curso de profesionalización docente por lo cual para el profesor, a esta altura de nuestras interacciones, era natural reflexionar sobre el saber matemático.
} 
directa y el profesor contestó porque se suma siempre 2.5, lo cual evidencia un pensamiento proporcional del tipo aditivo simple. Entonces, se le colocó el valor de 1.5 en el dominio y se le pidió que diera una explicación para ese valor. En ese momento, el modelo de pensamiento que el docente utilizaba no le era suficiente para argumentar su respuesta (ni la reducción a la unidad, ni el pensamiento aditivo simple). Esta pregunta es una evidencia de la psme que se realiza junto al docente. Posterior a ello, se discute que la proporcionalidad es un tipo de relación entre las magnitudes que se mantiene constante. Al buscar los tipos de relaciones posibles, se llega a la conclusión de que es la razón entre ambas magnitudes la que se mantiene constante (como hemos visto en su dimensión epistemológica de nuestra unidad socioepistémica). A continuación, retomamos la idea de la función $y=-x$ y nuevamente preguntamos si era de proporcionalidad directa o inversa. Al realizar la tabla, el docente se da cuenta de su propio error y se corrige, diciendo que es una función de proporcionalidad directa y que la constante es -1 porque siempre $d a-1$.

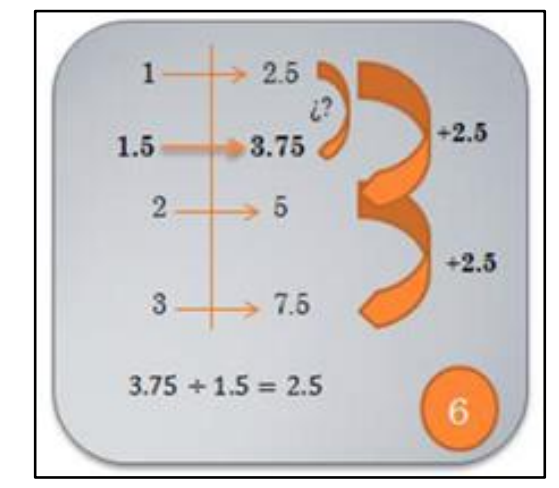

Figura 3 - Interacción 1 con el docente Fuente: desarrollado por los autores

Entonces, ¿qué sucedió hasta aquí? Por un lado, el docente profundizó en el desarrollo del pensamiento proporcional a partir de la psme. Por el otro, el docente vivenció la necesidad de conocer y reconocer distintas argumentaciones para un mismo saber matemático.

- Episodio comprobar la proporcionalidad

Al ingresar a clase con otro grupo de estudiantes luego de la psme, el docente planteó la misma actividad sobre la velocidad y tuvo la siguiente interacción con una de las estudiantes:

[10] Prof: Y para comprobar, comprobar qué hay proporcionalidad ahí... ¿cómo le podríamos hacer? ¿Cómo podríamos verificar?

[11] Sofía: Con una tabla... con una gráfica...

[12] Prof: A ver, permíteme (el docente se acerca al pizarrón y dibuja la tabla, encerrando a los números que E1 había colocado allí). Dónde o cómo presientes que esto... bueno ya me dices que esto es una tabla, la tabla ¿Del qué?

[13] Sofía: Del tres 
[14] Prof: (el profesor, en el pizarrón con E1, toma el plumón y comienza anotar) Del tres... Este valor y este que está aqui $(3,1)$, este valor y este que está aqui $(6,2)$, ¿Cómo podemos decir que son ... que hay una proporcionalidad, dame una justificación, qué otra forma? ¿Cómo podremos comprobar esa proporcionalidad? [15] Sofía: Dividiendo

[16] Prof: Ok, ¿qué valor y qué valor vas a dividir?

[17] Sofía: Voy a dividir 3 entre 1 y da igual a 3; 6 entre 2, me da igual a 3; si divido 9 entre 3 me da igual a 3 y 12 entre 4 da igual a 3 y así, todos me tienen que dar 3.

[18] Prof: Y eso ¿qué me indicará?

[19] Sofía: Que es la tabla del 3

[20] Prof: Eso que acabas de hacer tú, eso exactamente la relación ¿qué? La relación que estableció ella, entre estos dos, entre estos dos, entre estos dos (señala los pares ordenados)... y aquí, aqui la tienen (señala los resultados de las divisiones que daban 3) sale el mismo valor, ¿sí? Y por esa simple y sencilla razón...

[21] Estudiantes: Proporcionales.

Esta nueva interacción con la estudiante, más allá del análisis didáctico que pueda hacerse de la misma, nos enfocamos en la relación al saber matemático por parte del docente. Si bien no se muestra aquí por cuestiones de espacio (ver REYES-GASPERINI, 2011), durante el desarrollo de esta clase el docente reconoce y trabaja con pensamientos proporcionales del tipo multiplicativo, aditivo simple e inter. Al llegar a esta interacción, dada la respuesta de la estudiante, el docente comienza a tener interacciones didácticas con ella haciendo preguntas que promuevan el desarrollo del pensamiento proporcional de tipo intra. Esto quiere decir que no sólo lo reconoce sino que también lo promueve, por tanto, es privilegiada la articulación de distintas argumentaciones, permitiendo la emergencia de diversas racionalidades, en otras palabras, reconocer la emergencia de diferentes maneras de pensar una misma actividad. Asimismo, el desarrollo del carácter funcional del saber se evidencia a partir de relacionarse al saber matemático de la proporcionalidad como la relación entre las magnitudes que se mantiene constante, más allá de la centración en la algoritmetización de un conocimiento, como es el caso de la reducción a la unidad en nuestro ejemplo.

\section{Conclusiones}

Con la propuesta del empoderamiento docente atendemos a la profesionalización docente desde la problematización del saber matemático (psm) y despersonificamos el problema de investigación, ya no buscamos causas o responsables del fracaso, sino que ponemos el foco en el propio saber matemático, tanto al nivel de su significado como de su uso, y, desde allí, proporcionamos una alternativa viable. Nuestra propuesta, entonces, no 
provee de manera explícita estrategias didácticas o reflexiones pedagógicas sobre la enseñanza de la matemática; sino, más bien, hace del saber matemático escolar un problema y enmarca sobre él un conjunto de cuestionamientos que se trabajan con los docentes, quienes localizan y analizan los usos y razón de ser del saber matemático de lo proporcional, con base en la unidad de análisis socioepistémica construida.

En la investigación, la psm fue el cimiento de dicha unidad. Permitió, desde una mirada social y epistemológica del saber matemático, el reconocimiento de la dimensión práxica, funcional, situacional e histórica que está al nivel de la actividad de dicho saber, esto es: ante la imposibilidad de medir cualquier magnitud, dado que no existe una unidad común necesaria para la medición entre las dos magnitudes, se comparan, se construyen relaciones entre ellas y se busca aquella que se mantenga constante. Entonces, bajo la concepción de que las prácticas sociales son aquellas que norman intangiblemente la forma de construir conocimiento de los individuos, es decir, nos hacen hacer lo que hacemos, y por tanto están en la base de toda construcción del conocimiento, es natural que, en mayor o menor tiempo y medida, todos los individuos construyamos la noción de la proporcionalidad basándonos en lo que en sus orígenes motivó su construcción: la relación constante a partir de la comparación entre magnitudes, como lo hace el docente durante la psme.

En cuanto a su nivel cognitivo, el reconocimiento de los distintos modelos de pensamiento proporcional y su evolución, permitió distinguir los modelos que el docente desarrollaba en sus respuestas y cuestionar de manera tal que se desarrollen nuevos. Por último, bajo una dimensión didáctica, evidenciamos cómo el docente, al modificar su relación al saber matemático, genera interacciones didácticas, de naturaleza dialéctica, con los estudiantes en las cuales reconoce las distintas argumentaciones e incentiva, mediante cuestionamientos oportunos, el desarrollo del pensamiento proporcional hasta llegar al modelo intra, que se vincula con lo que recién hemos mencionado sobre la relación entre magnitudes. Por tanto, se evidenció en esta investigación que el proceso que debe vivir el saber matemático para hacer viable que el aprendizaje esté centrado en prácticas y no en objetos ajenos a la realidad (como postula el $d M E$ ) radica en la psm.

Asimismo, la psme con el docente permitió al docente hacerse dueño de una práctica en un sentido particular: el docente, luego de transitar una interacción entre colegas profesores e investigadores en un proceso de profesionalización y posteriormente su interacción durante el transcurso del estudio, desde su propia convicción ya que nosotros no aportamos sobre las cuestiones didácticas-pedagógicas de manera explícita, consolidó en la acción los cuestionamientos al saber matemático realizados en la psme, y, así, transformó su realidad 
áulica. Es decir, el docente se encuentra en una inmersión al proceso de empoderamiento docente.

Por tanto, postulamos al empoderamiento como el proceso vivido por el docente, en conjunto con sus colegas (profesores e investigadores) con objeto de comprender, asimilar, asumir, aceptar y adherirse a una propuesta novedosa sobre el aprendizaje centrado en prácticas y no en objetos abstractos, donde se privilegie la articulación de distintas argumentaciones, se permita la emergencia de diversas racionalidades situadas o contextualizadas, se posea o desarrolle el carácter funcional del saber, se favorezca la resignificación progresiva al considerar varios marcos de referencia, sobre la consideración de que las prácticas sociales son la base de la construcción que realizará el individuo del conocimiento matemático.

Como resultado de esta investigación postulamos como hipótesis que, por un lado, una propuesta de innovación para los diseños de formación docente debe sustentarse en la psme más que en la producción de prototipos de herramientas didácticas para el aula; por el otro, que la construcción de una unidad socioepistémica con base en la psm, desde un estudio socioepistemológico, permitirá en un futuro evaluar la incorporación a un proceso de empoderamiento docente considerando a la psme como punto de partida; y, por último, postulamos al empoderamiento docente como un mecanismo para atender al fenómeno de exclusión de la construcción del conocimiento matemático provocado por el $d M E$ (SOTO, 2010; SOTO; CANTORAL, en prensa) que debe acompañar el rediseño e inserción en el sistema educativo de este último. Entonces, como dijimos al comienzo, partimos del reconocimiento del docente como intelectual profesional que se ocupa de la formación académica, ética y ciudadana de la juventud de nuestra sociedad, por lo cual, atender a la profesionalización docente desde la psm, mediante el empoderamiento docente, despersonifica el problema y pone la mirada sobre lo que debemos atender: el rediseño del $d M E$. Así, habremos dado un paso más hacia la democratización del aprendizaje.

\section{Agradecimientos}

Agradecemos a los revisores y al equipo editorial de Bolema por el trabajo realizado durante el proceso de evaluación de nuestra propuesta. 


\section{Referencias}

BALL, D., THAMES, M.; PHELPS, G. Content Knowledge for Teaching: What Makes it Special? Journal of Teacher Education, Reston, VA, v. 59, n. 5, p. 389-407, nov./dic. 2008.

GELLERT, U.; BECERRA, R.; CHAPMAN, O. Research Methods in Mathematics Teacher Education. In: CLEMENTS, M. A.; BISHOP, A. J.; KEITEL-KREIDT, C.; KILPATRICK, J.; LEUNG, F. K. S. (Ed.). Third International Handbook of Mathematics Education. New York: Springer International. 2013. p. 327-360.

BEN-CHAIM, D.; ILANY, B. S.; KERET, Y. “Atividades Investigativas Autênticas” para o Ensino de Razão e Proporção na Formação de Professores de Matemática para os Níveis Elementar e Médio. Bolema, Rio Claro, Brasil, v. 21, n. 31, p. 125-159. 2008.

BLOCK, D.; GARCÍA, S. Fractal 1. Matemáticas. Secundaria. Primer grado. Distrito Federal: SM Ediciones, 2009.

CAMACHO, A. L. Reflexiones preliminares en torno al empoderamiento. 2003. Disponível em: <http://www.alforja.or.cr/centros/cep/documentos/poderyempoderamiento.pdf >. Acesso em: 20 nov. 2010.

CANTORAL, R. La aproximación socioepistemológica a la investigación en matemática educativa: una mirada emergente. In: CONFERENCIA INTERAMERICANA DE EDUCAÇÃO MATEMÁTICA - EDUCACIÓN MATEMÁTICA \& DESAFÍOS Y PERSPECTIVAS, 10., 2003, Blumenau. Anais... Blumenau: Universidad Regional de Blumenau, 2003. p. 1-15. CD-ROM

CANTORAL, R. Fundamentos y Métodos de la Socioepistemología. In: SIMPOSIO EN MATEMÁTICA EDUCATIVA, 1., 2011, Distrito Federal. Actas... Distrito Federal, México: CICATA del IPN. 2011. Disponível em: 〈http://www.youtube. com/watch?v=byHKKFnAq5Y> Acesso em: 12 maio 2013.

CANTORAL, R. Tendencias: Los métodos de investigación para profesionalización docente en Matemáticas. Revista Latinoamericana de Investigación en Matemática Educativa, México, D.F., v. 16, n. 1, p. 5-12, mar. 2013a.

CANTORAL, R. Teoría Socioepistemológica de la Matemática Educativa. Estudios sobre construcción social del conocimiento. Barcelona, España: Gedisa, 2013 b.

CANTORAL, R., FARFÁN, R. Pensamiento y lenguaje variacional en la introducción al análisis. Epsilon, Sevilla, v. 42, n. 14.3, p. 353-369. 1998.

CANTORAL, R.; REYES-GASPERINI, D. Matemáticas y Práctica social: Construcción social del conocimiento matemático. Novedades educativas, Buenos Aires, n. 261, p. 60-65, nov. 2012.

CANTORAL, R.; TUYUB, I.; Construcción social del conocimiento matemático durante la obtención de genes en una práctica toxicológica. Bolema, Rio Claro, Brasil, v. 26, n. 42a, p. 331-328, abr. 2012.

CARRETERO, L. La adquisición de la noción de proporcionalidad según diferentes tipos de estructuras multiplicativas por el niño de 8 a 11 años. Anuario de Psicología, Barcelona, v. 42, n. 3, p. 85-101. 1989.

CHEVALLARD, Y. El análisis de las prácticas docentes en la teoría antropológica de lo didáctico (trad. Ricardo Barroso Campos). Recherches en Didactique des Mathématiques, Grenoble, v. 19, n. 2, p. 221-266. 1999. 
CLIMENT, N.; ROMERO CORTÉS, J.; CARRILLO, J.; MUÑÓZ CATALÁN, M.; CONTRERAS, L. ¿Qué conocimientos y concepciones movilizan futuros maestros analizando un vídeo de aula? Revista Latinoamericana de Investigación en Matemática Educativa, México, D.F., v. 16, n. 1, p. 13-36, mar. 2013.

CONTRERAS, L.; CARRILLO, J.; ZAKARYAN, D.; MUÑOZ-CATALÁN, M.C.; CLIMENT, N. Un estudio exploratorio sobre las competencias numéricas de los estudiantes para maestro. Bolema, Rio Claro, Brasil, v. 26, n. 42b, p. 433-457, abr. 2012.

COVIÁN, O. El papel del conocimiento matemático en la construcción de la vivienda tradicional: El caso de la Cultura Maya. 2005. 192 f. Dissertação (Mestrado em Matemática Educativa) - Centro de Investigación y de Estudios Avanzados del IPN, México, 2005.

DA PONTE, J. P.; QUARESMA, M.; BRANCO, N. Práticas profissionais dos professores de matemática. Avances de Investigación en Educación Matemática, España, v. 1, n. 1, p. 65-86, maio 2012.

EUCLIDES. Elementos. Libros I-IV (Trad. por M. L. Puertas Castaños). Madrid: Gredos, 1991.

GODINO, J. D.; BATANERO, C. Proporcionalidad y su didáctica para maestros. Granada: Proyecto de Investigación y Desarrollo del Ministerio de Ciencia y Tecnología, 2002.

HOWE, A. C.; STUBBS, H. S. Empowering Science Teachers: A Model for Professional Development. Science Teacher Education, New Jersey, v. 8, n. 3, p. 167-182, Aug. 1998.

HOWE, A. C.; STUBBS, H. S. From Science Teacher to Teacher Leader: Leadership Development as Meaning Making in a Community of Practice. Science Teacher Education, New Jersey, v. 87, n. 2, p. 281-297, mar. 2003.s

INHELDER, B.; PIAGET, J. El equilibrio de la balanza. En: INHELDER, B.; PIAGET, J. (Ed.). De la lógica del niño a la lógica del adolescente. Ensayo sobre la construcción de las estructuras operatorias formales. Buenos Aires: Paidós, 1972. p. 142-155.

ITURBE, A.; RUIZ, M. E. Modos de acción y decisiones de los docentes. Un ejemplo en la enseñanza de la proporcionalidad. En: REUNIÓN LATINOAMERICANA DE MATEMÁTICA EDUCATIVA, 14., 2010, Guatemala. Actas... Guatemala, DF: Clame, 2011. p. 1047-1054.

LEZAMA, J. Una mirada socioepistemológica al fenómeno de la reproducibilidad. Revista Latinoamericana de Investigación en Matemática Educativa, México, D.F., v. 8, n. 3, p. 339-362, nov. 2005.

LLINARES, S.; VALLS, J.; ROIG, A-I. Aprendizaje y diseño de entornos de aprendizaje basado en videos en los programas de formación de profesores de matemáticas. Educación Matemática, México, D.F., v. 20, n. 3, p. 59-82, dic. 2008.

MARTÍN MARURI, I. Empoderamiento para la innovación social. En: CIBERVOLUNTARIOS. (Ed.). Innovación para el empoderamiento ciudadano a través de las TIC, 2011. p. 129-136. Disponível em: 〈http://www.empodera.org/pdf/libro.pdf>. Acceso em: 20 nov. 2011.

MARTÍNEZ, N. Y.; GONZÁLEZ, J. Construcción y uso significativo del concepto de proporcionalidad. Diseño e implementación de actividades desde la experiencia de investigación acción. In: ENCUENTRO COLOMBIANO DE MATEMÁTICA EDUCATIVA, 9., 2008, Valledupar. Actas... Valledupar: $\quad$ ASOCOLME. $2008 . \quad$ Disponible es: 〈http://funes.uniandes.edu.co/991/1/30Taller.pdf〉. Acceso en: 20 abr. 2010. 
MONTERO, M. Teoría y práctica de la psicología comunitaria. La tensión entre comunidad y sociedad. 3. ed. Buenos Aires: Paidós, 2006.

MONTIEL, G.; JÁCOME, G. Significado trigonométrico en el profesor. Bolema, Rio Claro, Brasil, en prensa.

OBANDO, G.; VASCO, C.; ARBOLEDA, L. Enseñanza y aprendizaje de la razón, la proporción y la proporcionalidad: un estado del arte. Revista Latinoamericana de Investigación en Matemática Educativa, México, D.F., v. 17, n. 1, p. 59-81, mar. 2014. doi: 10.12802/relime.13.1713

OLIVEIRA, I. Proporcionalidade: estratégias utilizadas na Resolução de Problemas por alunos do Ensino Fundamental no Quebec. Bolema, Rio Claro, Brasil, v. 22, n. 34, p. 57-80, 2009.

OLIVEIRA ARAMAN, E.; BATISTA, I. Contribuições da História da Matemática para a Construção dos Saberes do Professor de Matemática. Bolema, Rio Claro, Brasil, v. 27, n. 45, p. 1-30, abr. 2013.

OLLER, A.; GAIRÍN SALLÁN, J. La génesis histórica de los conceptos de razón y proporción y su posterior aritmetización. Revista Latinoamericana de Investigación en Matemática Educativa, México, D.F., v. 16, n. 3, p. 317-338, nov. 2013. doi: 10.12802/relime.13.1632

PARADA, R.; PLUVINAGE, F. Reflexiones de profesores de matemáticas sobre aspectos relacionados con su pensamiento didáctico. Revista Latinoamericana de Investigación en Matemática Educativa, México, D.F., v. 17, n. 1, p. 83-113, mar. 2014. doi: 10.12802/relime.13.1714

PIAGET, J.; INHELDER, B. El preadolescente y las operaciones proposicionales. En: PIAGET, J.; INHELDER, B. (Ed.). Psicología del niño. 7. ed. Madrid: Ediciones Morata, 1977. p. 131-150.

PISANO, J. P. Logikamente. Título del tema: Regla de Tres simple. Número de tema: 02. Área: Matemática. Buenos Aires: Ediciones Logikamente, 2011.

REYES, D. Empoderamiento docente desde una visión Socioepistemológica: Estudio de los factores de cambio en las prácticas del profesor de matemáticas. 2011. 200 f. Dissertação (Mestrado em Matemática Educativa) - Centro de Investigación y de Estudios Avanzados del IPN, México, 2011.

REYES-GASPERINI, D. Reflexiones acerca del aula actual, como desafío para el profesor de matemática. Premisa, Buenos Aires, v. 12, n. 44, p. 44-50, feb. 2010.

REYES-GASPERINI, D.; CRESPO CRESPO, C. Un estudio acerca del fenómeno de exclusión a nivel superior en la carrera de profesorado de matemática. En: REUNIÓN LATINOAMERICANA DE MATEMÁTICA EDUCATIVA, 24., 2010, Guatemala. Actas... Guatemala, DF: Clame, 2011.p. 897904.

RIVAS, M.; GODINO, J. D; CASTRO, W. Desarrollo del Conocimiento para la Enseñanza de la Proporcionalidad en Futuros Profesores de Primaria. Bolema, Rio Claro, Brasil, v. 26, n. 42b, p. 559588, abr. 2012.

SALAZAR, M.; DÍAZ, L. La actividad de medir aporta significados a fracciones y razones. En: REUNIÓN LATINOAMERICANA DE MATEMÁTICA EDUCATIVA, 22., 2008, Distrito Federal. Actas... Distrito Federal: Clame, 2009. p. 207-216. 
SÁNCHEZ ORDOÑEZ, E. Razones, proporciones y proporcionalidad en una situación de reparto: Una mirada desde la Teoría Antropológica de lo Didáctico. Revista Latinoamericana de Investigación en Matemática Educativa, Distrito Federal, México, v. 16, n. 1, p. 65-97, mar. 2013.

SILVA DREYER, C. L.; MARTÍNEZ GUZMÁN, M. L. Empoderamiento, Participación y Autoconcepto de Persona Socialmente Comprometida en Adolescentes Chilenos. Interamerican Journal of Psychology, San Juan, v. 41, n. 2, p. 129-138, 2007.

SILVA-CROCCI, A.; CORDERO OSORIO, F. Matemática Educativa, identidad y Latinoamérica: el quehacer y la usanza del conocimiento disciplinar. Revista Latinoamericana de Investigación en Matemática Educativa, México, D.F., v. 15, n. 3, p. 295-318, nov. 2012.

SOTO, D. El Discurso Matemático Escolar y la Exclusión. Una Visión Socioepistemológica. 2010. 105 f. Dissertação (Mestrado em Matemática Educativa) - Centro de Investigación y de Estudios Avanzados del IPN, México, 2010.

SOTO, D.; CANTORAL, R. El discurso Matemático Escolar y la Exclusión. Una visión Socioepistemologica. Bolema, Rio Claro, Brasil, en prensa.

STOLK, M. J.; DE JONG, O.; BULTE, A. M. W.; PILOT, A. Exploring a Framework for Professional Development in Curriculum Innovation: Empowering Teachers for Designing Context-Based Chemistry Education. Research in Science Education, Grenoble, v. 41, n. 3, p. 369-388, 2011.

VALDEMOROS, M. Dificultades didácticas en la enseñanza de razón y proporción: estudio de caso. En: REUNIÓN LATINOAMERICANA DE MATEMÁTICA EDUCATIVA, 23., 2009, Santo Domingo. Actas... Santo Domingo: Clame, 2010. p. 217-226.

VERGNAUD, G. La teoría de los campos conceptuales. Recherchers en Didactiques des Mathématiques, Grenoble, v. 10, n. 2, p. 133-170, 1990. 